Original Article

\title{
A study to assess the knowledge and practice of fast food consumption among Pre-University students in Udupi Taluk, Karnataka, India
}

\author{
Tenneychell Khongrangjem, Sushma Marita Dsouza*, Priya Prabhu, \\ Vijay Basappa Dhange, Vrindha Pari, Sunil Kumar Ahirwar, Kumar Sumit
}

Department of Public Health, Manipal University, Manipal, India

\section{A R T I C L E I N F O}

\section{Article history:}

Received 9 September 2017

Accepted 21 November 2017

Available online 22 November 2017

\section{Keywords:}

Fast food

Knowledge

Practice

Pre-University students

\begin{abstract}
A B S T R A C T
Introduction: Fast food is processed and prepared in an industrial fashion. Vitamins, minerals, fibre and amino acids are low or lacking in fast food but has high energy (calories).The present study was done to assess the knowledge and practice of fast food consumption among Pre-University College students. Materials and methods: The study design adopted was cross-sectional. Semi-structured self-administered questionnaire were used to collect the data. Time bound enumeration was used to recruit the participants and total 160 Pre-University students were included in the study.

Result: About 51(31.87\%) of the participants had inadequate knowledge, 67(41.88\%) of the participants had moderate knowledge and $42(26.25 \%)$ of the participants had adequate knowledge about the effect of fast food consumption. The majority of the respondents 116 (72.5\%) reported that the main reason for their consumption is a delicious taste of fast food.

Conclusion: Though fast foods are tasty they have low nutritive value and high calories. Nutrition counselling regarding the significance of a balanced diet and harmful effects of fast foods may help to curb the fast food addiction.
\end{abstract}

(C) 2017 INDIACLEN. Published by Elsevier, a division of RELX India, Pvt. Ltd. All rights reserved.

\section{Introduction}

Fast food indicates food which is quickly prepared and served at outlets such as fast food restaurants. ${ }^{1}$ Healthy nutrition rich foods have been substituted by the new food named as fast food. ${ }^{2}$ Fast food is made and processed in an industrial fashion, i.e., with standard ingredients, consistent cooking and production methods. ${ }^{1}$ Vitamins, minerals, fibre and amino acids are low or lacking in fast food, but have high energy (calories). In the circumstance of the global economy, fast food is a universal phenomenon. The availability of fast food at minimum prices and marketing approaches adopted by manufacturers of such foods has elicited a progression of fast food at high rate. Fast food does not contain the nutrients which are required to sustain our body health. Therefore these foods that have low nutritive value are considered injurious to health and may be named as junk food. ${ }^{2}$ Popularity of fast food in this era of growth has been attributed to speedy preparation and ease of finishing a food within no time. ${ }^{3}$

\footnotetext{
* Corresponding author.

E-mail addresses: sushmadsouza22@gmail.com, princialobo2000@gmail.com (S.M. Dsouza).
}

There is a rising trend among the newer generation about fast food culture. ${ }^{2}$ Elder people are more fascinated about healthy foods as compared to adolescents. ${ }^{4}$ The fast-food sector in India is rising at the rate of $40 \%$ per annum. India ranks 10 th in the fast food per capita expenditure data with $2.1 \%$ of spending in yearly over-all expenditure. ${ }^{2}$ The current universal adaptation of a system for fast food consumption had led to several harmful effects on health. ${ }^{5}$

Thus the low nutritive value and high calories of fast food causes obesity in children. Junk foods also contain colours which are often inedible, carcinogenic and injurious to the body. ${ }^{3}$ This fast food affects the digestive system and its effects can occur after several years. ${ }^{6}$ Fast food is a rising factor in the diet and since 1970s the rate of fast food use has grown intensely. Fast food is particularly common among adolescents, who often go to fast food stalls twice per week. Nutritional requirements in proper proportions, particularly in the younger age group assume a pivotal role in the overall development process. Medically it has been evidenced that the nutritional rate of fast food is less than the required amount and its consumption leads to several disorders. Also, the fast foods are key agents causing various disorders and diseases like obesity ${ }^{7}$ which is likely to cause heart diseases in the future. ${ }^{1}, 8$ College students are vastly exposed to harmful eating practices causing increased body weight. ${ }^{9}$ Hence the current study was conducted to assess the knowledge and practice of adolescents 
regarding fast food consumption, which may be helpful to prepare a nutritional policy to improve the nutritional status of adolescents.

\section{Materials and Methods}

\subsection{Study design, setting and sample size}

A cross-sectional study was conducted from August to November 2016 among Pre-University students. The study was conducted in two Pre-University Colleges in Udupi district, Karnataka. Since, we got permission from only two Pre- University Colleges and the study period was four months we used time bound enumeration to recruit the participants. Prior permission was taken from the college authorities and depending on the dates given, the study had been carried out. The study was carried out before the commencement of the exams and 160 participants were included in the study. No one refused to take part in the study. There was an active participation from all the students.

\subsection{Inclusion criteria and Exclusion criteria}

Pre-University College students from Udupi Taluk and students who were willing to take part in the study were included in the study. Students those who were absent on the day of data collection were excluded from the study.

\subsection{Data Collection Methods}

Data was obtained from the participants using a semistructured self-administered questionnaire which included details on socio-demographics of the participants, knowledge and practice of fast food consumption among participants. The questionnaires were formulated based on knowledge and practice of the college students regarding fast food consumption. The questionnaires were prepared based on the local context with regard to the different articles on the same topic of interest. The questionnaires were validated by expert opinion and it was pilot tested. Questionnaires were administered in both Kannada and English languages. The participants had to answer the ten questions to assess their knowledge regarding fast food. Every right answer gained one (1) point and every wrong answer gained zero (0). The overall score ranged from 0-10. Participants with scores $0-3$ were considered to have inadequate knowledge, those with 4-7 points had moderate knowledge while those with 8-10 points had adequate knowledge. The participants took approximately $15-20 \mathrm{~min}$ to complete the questionnaire.

\subsection{Analysis}

The Statistical Package for Social Sciences 20(SPSS 20) for Windows was used to analyze the data. Through descriptive statistics, socio-demographic, knowledge of the participants were described and expressed as frequencies and percentages. To compare the knowledge among males and females Chi square test was employed. P value $(<0.05)$ was considered statistically significant.

\subsection{Ethical Consideration}

Through Institutional Ethics Committee (IEC) (IEC 580/2016) ethical clearance was obtained. Written informed consent was obtained from all the participants after the purpose of the study was explained to them using a participant information sheet and participation in the study was voluntary. Parental consent was not obtained for participants $<18$ yrs of age but permission was sought from the college authorities.

Confidentiality of the data was guaranteed.

\section{Results}

Table 1 describes about the socio-demographic profile of the Pre-University students. The study included males 83 (51.9\%) and females $77(48.1 \%)$ respectively. With respect to religion, maximum $118(73.8 \%)$ number of the participants followed the Hindu religion. More than half of the respondents were from second PUC class i.e., 90 (56.25\%) and 95 (59.37\%) participants were living in rural areas. With respect to monthly family income, 77 (48.13\%) participants had family income of 10000-20000.

Table 2 describes about practice of fast food consumption among the study participants .In this study 15(9.4\%) of the participants reported that they eat fast food regularly and 115 (71.8\%) reported that sometimes they had fast food. Based on frequency of fast food consumption, 67 (41.9\%) reported once in a month and the majority of the participants 100 (62.5\%) reported that they had Chines food. With regard to quantity, 102 (66.2\%) eat a medium amount of fast food and 83 (51.9\%) reported they drank soft drinks. The majority of the respondents 116 (72.5\%) reported that the main reason to have fast food was because of its delicious taste.

Table 3 describes that 51(31.87\%) of participants had inadequate knowledge, 67(41.88\%) of the participants had moderate knowledge and $42(26.25 \%)$ of the participants had adequate knowledge about the effects of fast food consumption.

The difference of knowledge between males and females was statistically significant $(\mathrm{P}<0.01)$. Out of 83 males, $39(47 \%)$ had inadequate knowledge, 27 (32.5\%) had moderate knowledge and $17(20.5 \%)$ had adequate knowledge. Out of 77 females, $12(15.6 \%)$ had inadequate knowledge, 40(51.9\%) had moderate knowledge and 25 (32.5\%) had adequate knowledge.

\section{Discussion}

A study to assess the knowledge and practice fast food consumption among Pre-University students was conducted in Udupi Taluk of Karnataka, India. Total 160 participants were involved in the study.

About 1/4th of the study participants consumed fast food on an average of two to four times per week. ${ }^{10}$ Another study found that $40.1 \%$ of the students consumed fast food rarely and $8.0 \%$ of the students consumed fast food everyday. ${ }^{11}$ In the present study 67 (41.9\%) participants reported the consumption of fast food for once

Table 1

Distribution of respondents on the basis of Socio-demographic characteristics $(\mathrm{n}=160)$.

\begin{tabular}{llll}
\hline Socio-demographic Characteristics & & Frequency & Percentage \\
\hline Gender & Male & 83 & 51.9 \\
& Female & 77 & 48.1 \\
Age & $15-18$ & 158 & 98.8 \\
& $19-21$ & 2 & 1.2 \\
Religion & Hindu & 118 & 73.8 \\
& Muslim & 12 & 7.5 \\
\multirow{3}{*}{ Educational Status } & Christian & 30 & 18.7 \\
\multirow{2}{*}{ Place of Residence } & 1st PUC & 70 & 43.8 \\
& 2nd PUC & 90 & 56.2 \\
Monthly Family Income (In Rupees) & Urban & 65 & 40.6 \\
& Rural & 95 & 59.4 \\
& $10,000-20,00$ & 42 & 26.3 \\
& $>20,000$ & 41 & 48.1 \\
\hline
\end{tabular}

$\mathrm{n}=$ Number. 
Table 2

Distribution of respondents based on practice of Fast Food Consumption $(n=160)$.

\begin{tabular}{|c|c|c|c|}
\hline The types of fast food and the factors affecting it & & Frequency & Percentage \\
\hline \multirow[t]{3}{*}{ Eat restaurant fast food regularly? } & Yes & 15 & 9.4 \\
\hline & No & 30 & 18.8 \\
\hline & Sometimes & 115 & 71.8 \\
\hline \multirow[t]{3}{*}{ How often do you eat fast food? } & Once a month & 67 & 41.9 \\
\hline & Once a week & 63 & 39.4 \\
\hline & Twice a week & 30 & 18.7 \\
\hline \multirow[t]{5}{*}{ What kind of fast foods do you eat? } & Pastries & 9 & 5.6 \\
\hline & Pizza & 18 & 11.2 \\
\hline & French Fries & 13 & 8.2 \\
\hline & Cheese Items & 20 & 12.5 \\
\hline & Chinese Food & 100 & 62.5 \\
\hline \multirow[t]{3}{*}{ Portion size of fast food do you usually eat } & Small & 49 & 30.6 \\
\hline & Medium & 106 & 66.2 \\
\hline & Large & 5 & 3.2 \\
\hline \multirow[t]{5}{*}{ Kind of beverages do you drink with fast food } & Soft drinks & 83 & 51.9 \\
\hline & Coffee/tea & 16 & 10.0 \\
\hline & fresh fruit juices & 47 & 29.3 \\
\hline & Sweetened fruit drinks & 8 & 5.0 \\
\hline & energy drinks & 6 & 3.8 \\
\hline \multirow[t]{5}{*}{ why do you eat fast food } & delicious taste & 116 & 72.5 \\
\hline & attractive advertisements & 11 & 6.9 \\
\hline & diversity of fast food types & 9 & 5.6 \\
\hline & Convenience & 10 & 6.2 \\
\hline & availability of fast food restaurants & 14 & 8.8 \\
\hline
\end{tabular}

Table 3

Distribution of respondents on the basis of Knowledge $(n=160)$.

\begin{tabular}{llll}
\hline Levels of Knowledge & & Frequency & Percentage \\
\hline Knowledge & Inadequate & 51 & 31.9 \\
& Moderate & 67 & 41.9 \\
& Adequate & 42 & 26.2 \\
\hline
\end{tabular}

in a month. Taste factor (57.6\%) and time factor (21.5\%) was one of the reasons to choose fast foods ${ }^{10}$. The majority of the participants revealed that the flavour or taste was the key attraction to have fast food. ${ }^{1}$ Proximity, numerous locations, lack of time and speed of service were reasons behind the preference of fast food by the American students, which was 70\% among males and 68\% among females. Fast food consumption was least among Spanish participants, i.e. $26 \%$ among females and $21 \%$ among males. ${ }^{12}$ The present study revealed that the main reason for the consumption of fast food among participants was a delicious taste of the food. Another study found that the key reasons for fast food consumption were: routine/desire (42.1\%), easy availability (30.5\%), dearth of other appropriate choice (13.7\%), and peer influence $(13.2 \%)^{13}$ In a cross-sectional study, $46.7 \%$ of the respondents indicated the fast food is not only easy to get but also fast. ${ }^{14}$ The reason for fast food consumption was that it does not require much time as it can be prepared easily. ${ }^{15}$ Another study included 349 adolescents; among them $35 \%$ of adolescents had fast food six or more times per week. The taste was the most important factor for $72 \%$ of adolescents to select their food. ${ }^{16}$ A cross-sectional study in Bangladesh revealed that out of 200 students' 197 (98.5\%) students reported their preference for junk food and around $22 \%$ of the participants revealed that they have fast food for four days per week whereas $21.3 \%$ reported to have fast food every day. ${ }^{13}$ Out of 265 students, $15.1 \%$ went to the fast food outlets every day, $19.6 \%$ once a week, $31.7 \%$ two times per week, $17.7 \%$ three times per week, $10.6 \%$ once in every two weeks and $5.3 \%$ once in a month. ${ }^{15}$ On special days and while meeting friends fast food consumption were (33.9\%) and $(25.7 \%)$ respectively. ${ }^{17}$

The order of preference for outside food stuff was chat items (42\%), pizza (23\%), fast foods (19\%) and noodles (16\%) which were in the top category of preference. ${ }^{10}$ The kind of fast food chosen by the students included pizzas, fried chicken, noodles, burgers, and soft drinks. ${ }^{13}$ Factors such as food quality, convenience, decoration, atmosphere, speediness of service, variety in menu and availability of special products play an important role with respect to young people's preference to fast food outlets. Among the fast food products, the hamburger was the first to come to the minds of $76.2 \%$ participants followed by pizza $7.5 \%$, toasted sandwich $6.4 \%$, biscuits $4.2 \%$, chips $3.4 \%$, sandwich $1.5 \%$ and roll $0.8 \%{ }^{15}$ In the present study maximum participants 100 (62.5\%) reported that they had Chines food.

The half of the study population (50\%) was aware about the adverse effects of the junk food. ${ }^{10}$ Another study revealed that $69 \%$ of adolescents had moderate knowledge regarding effects of fast food consumption on health. ${ }^{16}$ About 98\% (196) students were worried about the harmful effects related to fast food consumption and almost $80 \%$ students aware about the ingredients of their fast food and $95.6 \%$ students knew that fast food comprises of huge amounts of fats and oils. ${ }^{13}$ In the current study 42 (26.25\%) of the participants had adequate knowledge about the effect of fast food consumption.

\section{Conclusion}

Good nutritional diet or balance diet is fundamental need for development and growth of human beings. Most of the students especially children and young adults during their meal time have fast food and get habituated to the taste of the fast food. The fast food consists anything that is delicious, quick, fashionable and convenient. Though fast foods are tasty they have poor nutritive value and high calories. Nutrition counselling regarding the significance of a balanced diet and harmful effects of fast foods may help to curb the fast food addiction.

\subsection{Strengths}

The present study targeted the adolescents as they are vastly exposed to harmful eating practices. So it may be useful to prepare a nutritional policy to improve the nutritional status of adolescents. 


\subsection{Limitation}

As time bound enumeration was used to recruit the participants, generalization of the study was limited to the sample.

\subsection{Recommendations}

We advise to conduct the study along with awareness campaign regarding fast food and balanced diet so that students would be aware of harmful effects of fast food and usefulness of balanced diet.

\section{Acknowledgement}

The authors would like to express thanks to all the students who participated in this study. We would like to show gratitude to the Department of Public Health, Manipal University, Manipal, Manipal Pre-University College, Manipal and Mahatma Gandhi Memorial PU College, Udupi for their support. The authors declare no funding and no conflicts of interest.

\section{References}

1. Vaida N. Prevalence of fast food intake among urban adolescent students. IJES. 2013;2(1):353-359.

2. Ashakiran S, Deepthi R. Fast foods and their impact on health. JKIMSU. 2012;1 (2):7-15.

3. Pawar VK. Health hazards and knowledge about junk foods-a review. IJNR. 2015;1(2):146-157.
4. Moaadeli Z, Neyrizi AH, Sharifikia I, Marandi KA. The study of knowledge, attitude and nutritional practice of secondary school students in Darab City, Fars Province, Iran in 2011-2012. Int J School Health. 2015;2(2):1-7.

5. Meena M, Varma K. Fast food consumption among adolescent school girls in Jaipur. Int J Innov Res Rev. 2015;3(3):38-42.

6. Arya G, Mishra S. Effects of junk food \& beverages on adolescent's health - a review article. IOSR-INHS. 2013;1(6):26-32.

7. Antony M, Bhatti RK. Junk food consumption and knowledge about its ill effects among teenagers: a descriptive study. IJSR. 2015;4(6):1133-1136.

[8]. Steffi S, Josephine RM. A Case Study on Trend of Food Style among College Students. IJAPBC. 2013;2(1):103-107.

9. Al-Rethaiaa AS, Fahmy AA, Al-Shwaiyat NM. Obesity and eating habits among college students in Saudi Arabia: a cross sectional study. Nutr J. 2010;9(39):110.

10. Vidya B, Damayanthi MN, Sharada R, Manjunatha Shashikala. Junk food consumption pattern and obesity among school going children in an urban field practice area: a cross sectional study. J Evid Based Med Hlthcare. 2015;2 (12):1845-1851.

11. Yardimci H, Ozdogan Y, Ozcelik AO, Surucuoglu MS. Fast-food consumption habits of university students: the sample of Ankara. Pak J Nutr. 2012;11 (3):265-269.

12. Bryant R, Dundes L. Fast food perceptions: a pilot study of college students in Spain and the United States. Appetite. 2008;51:327-330.

13. Bipasha MS, Goon S. Fast food preferences and food habits among students of private universities in Bangladesh. South East Asia J Public Health. 2013;3 (1):61-64.

14. Jafnan R. Fast food consumption among local Australian students and international students in Australia. IJIR. 2017;3(3):983-989.

15. Onurlubas E, Yilmaz N. Fast food consumption habits of university students. $J$ Food Agric Environ. 2013;1(3\&4):12-14.

16. Saranya PV, Shanifa N, Susan S, Thomas SJU, Shetty AP. Adolescents' knowledge regarding the effects of fast food on health. Int J Curr Med Res. 2016;5(3):406409.

17. Seo H, Lee S, Nam S. Factors influencing fast food consumption behaviors of middle-school students in Seoul: an application of theory of planned behaviors. Nutr Res Pract. 2011;5(2):169-178. 